\title{
Malaria parasite liver stages render host hepatocytes susceptible to mitochondria-initiated apoptosis
}

\author{
A Kaushansky ${ }^{*, 1}$, PG Metzger ${ }^{1}$, AN Douglass ${ }^{1}$, SA Mikolajczak¹, V Lakshmanan, HS Kain ${ }^{1}$ and SHI Kappe ${ }^{*, 1,2}$
}

Intracellular eukaryotic parasites and their host cells constitute complex, coevolved cellular interaction systems that frequently cause disease. Among them, Plasmodium parasites cause a significant health burden in humans, killing up to one million people annually. To succeed in the mammalian host after transmission by mosquitoes, Plasmodium parasites must complete intracellular replication within hepatocytes and then release new infectious forms into the blood. Using Plasmodium yoelii rodent malaria parasites, we show that some liver stage (LS)-infected hepatocytes undergo apoptosis without external triggers, but the majority of infected cells do not, and can also resist Fas-mediated apoptosis. In contrast, apoptosis is dramatically increased in hepatocytes infected with attenuated parasites. Furthermore, we find that blocking total or mitochondria-initiated host cell apoptosis increases LS parasite burden in mice, suggesting that an anti-apoptotic host environment fosters parasite survival. Strikingly, although LS infection confers strong resistance to extrinsic host hepatocyte apoptosis, infected hepatocytes lose their ability to resist apoptosis when anti-apoptotic mitochondrial proteins are inhibited. This is demonstrated by our finding that B-cell lymphoma 2 family inhibitors preferentially induce apoptosis in LS-infected hepatocytes and significantly reduce LS parasite burden in mice. Thus, targeting critical points of susceptibility in the LS-infected host cell might provide new avenues for malaria prophylaxis.

Cell Death and Disease (2013) 4, e762; doi:10.1038/cddis.2013.286; published online 8 August 2013

Subject Category: Experimental Medicine

Plasmodium parasites, their mosquito vectors, and their host species have coevolved for millions of years. ${ }^{1}$ For humans, malaria is the most deadly parasitic infection in the world, with 350-500 million clinical episodes annually, mainly in the developing world. There is no effective vaccine in use, and attempts to control the disease with drugs alone have been plagued by the development of drug-resistant parasites. ${ }^{2}$ Parasites initiate asymptomatic infection in the liver, when mosquito-transmitted sporozoites invade hepatocytes. Ensconced within their host hepatocyte, they undergo rapid growth as liver stages (LS), exploiting host cell resources and ultimately produce tens of thousands of red blood cellinfectious forms, which are released from the hepatocyte and initiate symptomatic red blood cell infection. ${ }^{3}$ LS infection constitutes an insult to the infected hepatocyte that the host likely counters by initiating defense programs aimed at eliminating the parasite or the infected cell. To survive, parasites must be able to successfully control their host hepatocyte. During invasion, the sporozoite triggers the formation of a parasitophorous vacuole membrane (PVM) that encloses the parasite, and persists during subsequent intracellular LS development. The PVM is the major interface between the parasite and its hepatocyte host; yet, the importance of the PVM remains poorly characterized, including any role in countering hepatocyte defenses.

Previous work using rodent malaria models has shown that infected hepatocytes resist certain forms of artificial apoptotic stimuli in in vitro infection models, presumably a parasitecontrolled function to ensure LS survival. ${ }^{4,5}$ How these findings translate to infection in a host, and the extent to which LS parasites confer resistance to apoptosis during intrahepatocytic development, remains unexplored. As hepatocytes respond to apoptotic stimuli predominantly by utilizing mitochondria-dependent signaling, ${ }^{6,7}$ the LS parasite might have evolved to interfere with this property. Here we investigate the relationship between the malaria parasite LS infection and apoptosis of the hepatocyte host. Our results highlight the role that hepatocyte apoptosis has in curtailing parasite LS infection. In addition, we describe scenarios when the LS parasite is able to successfully mold its host cell environment in order to ensure survival.

\section{Results}

We developed a system to quantitatively compare rates of apoptosis in LS infected and uninfected cells in the same culture by building on previous work utilizing flow cytometry to monitor infection rates. ${ }^{8}$ Plasmodium yoelii-infected Hepa 1-6 cell cultures were stained with two antibodies, one specific to circumsporozoite protein (CSP) (Figure 1a; Supplementary Figure 1) and a second that recognizes cleaved forms of caspase-3, a committed step in apoptosis (Figure 1b; Supplementary Figure 1). To ensure that caspase-3 signal was specific, extrinsic apoptotic signaling was triggered by

\footnotetext{
${ }^{1}$ Seattle Biomedical Research Institute, Seattle, WA, USA and ${ }^{2}$ Department of Global Health, University of Washington, Seattle, WA, USA

${ }^{*}$ Corresponding author: A Kaushansky or SHI Kappe, Malaria Program, Seattle Biomedical Research Institute, 307 Westlake Avenue North Suite 500, Seattle, WA 98109, USA. Tel: + 206256 7216/7205; Fax: + 206256 7229; E-mail: alexis.kaushansky@seattlebiomed.org or stefan.kappe@ seattlebiomed.org

Keywords: Plasmodium; liver stage; apoptosis; cellular signaling

Abbreviations: LS, liver stage; CSP, circumsporozoite protein; PVM, parasitophorous vacuole membrane; Bcl-2, B-cell lymphoma 2

Received 28.2.13; revised 29.5.13; accepted 28.6.13; Edited by M Piacentini
} 
a

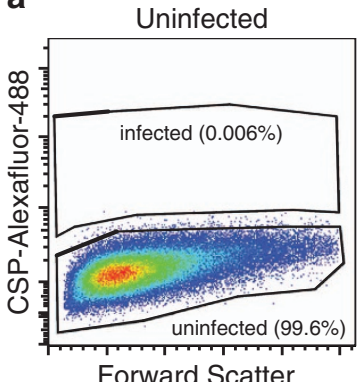

Forward Scatter

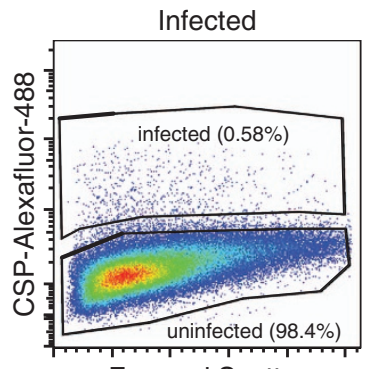

Forward Scatter b

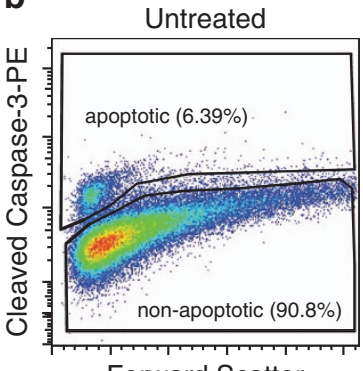

Forward Scatter

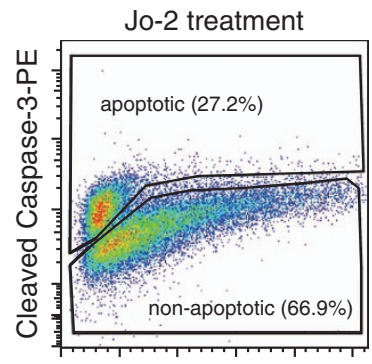

Forward Scatter
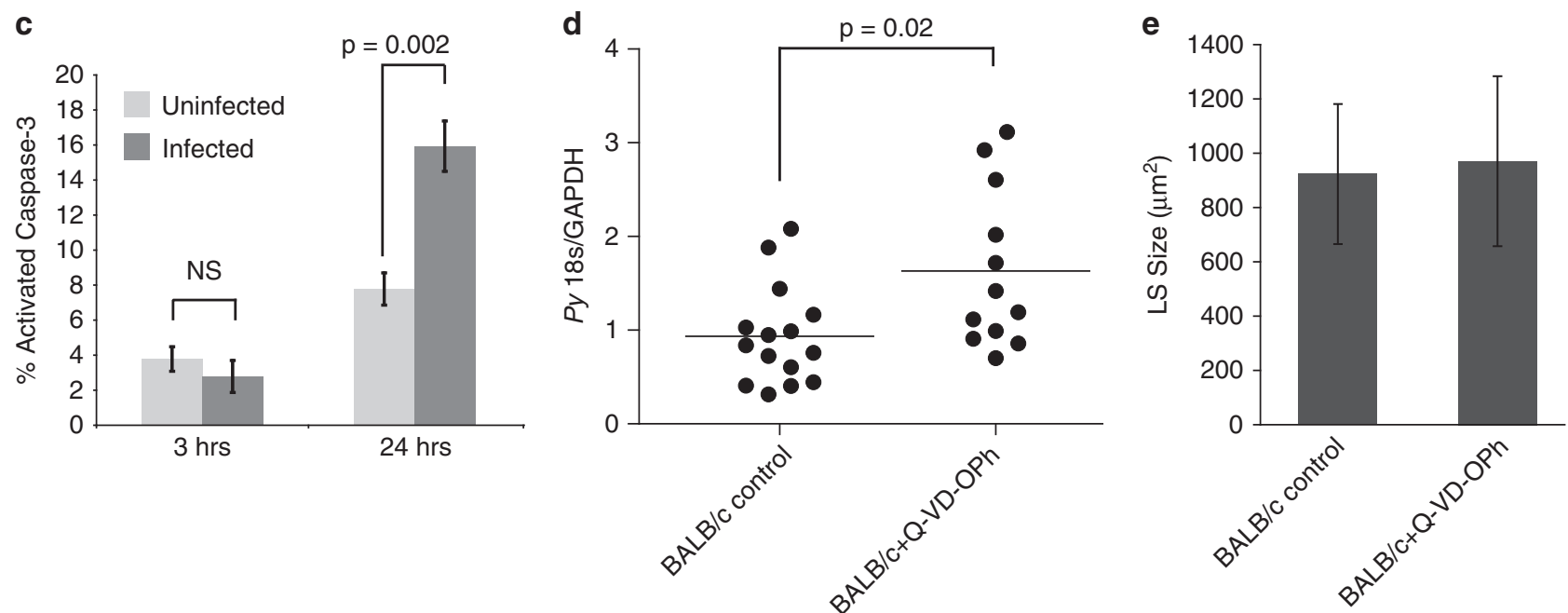

Figure 1 Apoptosis plays a role in clearing LS-infected hepatocytes in vitro and in vivo. (a) Cells are stained with an antibody against CSP. Gating strategy used for identifying infected cells is shown. (b) Cells are stained with an antibody against cleaved caspase-3. Gating strategy used for identifying apoptosis in infected or uninfected cells is shown. (c) Wild-type parasites elicit an apoptotic response in Hepa 1-6 cells 24, but not $3 \mathrm{~h}$ after infection. (d) BALB/cJ mice were treated with 20 mg/kg Q-VD-OPh beginning $1 \mathrm{~h}$ before infection, and then once daily. Mice were infected with $100000 \mathrm{P}$. yoelii sporozoites. LS burden was measured $42-44 \mathrm{~h}$ after infection by quantitative PCR. Q-VD-OPh-treated mice have significantly higher LS burden than untreated mice. (e) Parasites treated with $\mathrm{Q}-\mathrm{VD}-\mathrm{OPh}$ or control were measured $42 \mathrm{~h}$ after infection. There is not a significant difference in the size of parasites, indicating that Q-VD-OPh-treated mice harbor more parasites, not similar numbers of larger parasites

treating Hepa1-6 cells with a Fas-activating antibody (Jo-2). We observed significantly elevated levels of apoptosis both 6 and $24 \mathrm{~h}$ following treatment with Jo-2 (Figure 1b). This demonstrates that Hepa 1-6 cells respond to Fas activation, and that monitoring caspase- 3 cleavage in these cells unambiguously marks apoptosis.

A subset of LS-infected hepatocytes undergoes apoptosis. Surprisingly, untreated LS-infected cells more frequently exhibited elevated levels of apoptosis compared with untreated, uninfected cells $24 \mathrm{~h}$ after infection (Figure 1c; Supplementary Figure 1). This suggests that there is a subset of LS parasites that either cannot withstand the host defenses that trigger hepatocyte death or actively initiates apoptosis in their host cell. As hepatoma cells typically respond to apoptotic stimuli in different ways than primary hepatocytes, we tested the effect of host cell apoptosis on LS infection in vivo. BALB/cJ mice were treated with the pancaspase inhibitor Q-VD-OPh, ${ }^{9}$ infected with sporozoites, and parasite burden in the liver was measured after $44 \mathrm{~h}$. The LS burden nearly doubled in mice treated with Q-VD-OPh (Figure 1d). LS parasites were of similar size in treated and untreated mice (Figure 1e), indicating that the elevated level of LS burden observed was not due to increased growth but instead due to greater numbers of developing LS parasites. Taken together, these data suggest that under normal infection conditions in vivo, a subset of LS-infected hepatocytes die by caspase-dependent mechanisms without extrinsic pro-apoptotic stimuli. When host cell death is artificially blocked, these infected cells survive and allow parasites to develop.

Lack of a PVM induces apoptosis in LS-infected cells. It remains unknown whether impaired functions or fitness of the parasite could result in loss of apoptotic resistance in the infected host cell. It has been previously demonstrated that attenuated parasites that cannot complete LS development elicit apoptosis in infected hepatoma cells. ${ }^{10}$ When a viable malaria parasite productively invades a hepatocyte, it surrounds itself with a membrane of hepatocyte origin, called the PVM, which is subsequently modified by the LS parasite. This membrane serves as a critical interface between the parasite and its hepatocyte host. ${ }^{11,12}$ We hypothesized that the PVM might have a role in mediating resistance to host cell apoptosis after infection. We tested this by using transgenic parasites and markers that delineate parasites 
that have invaded with an intact PVM and those that reside inside the hepatocyte without a PVM.

Wild-type sporozoites can invade hepatocytes while forming a PVM (leading to productive infection) or enter by cell traversal, which is mediated by membrane wounding and does not form a PVM. ${ }^{13}$ Attenuated parasites that lack the 6-cys proteins P52 and P36 (p52(-)/p36(-)) enter hepatocytes but cannot form or maintain a $\mathrm{PVM}^{14}$ (Figure 2a). In contrast, parasites that lack the traversal protein SPECT2 can invade hepatocytes only during PVM formation (Supplementary Figure 2). Strikingly, when we infected cells with $P$. yoelii p52 $(-) p 36(-)$ parasites, we observed highly elevated levels of apoptosis in infected hepatocytes (Figure $2 \mathrm{~b}$ ) within $3 \mathrm{~h}$ of infection, which was not observed in $P$. yoelii wild-type-infected cells, suggesting that wild-type parasites have more robust control over their host cell. Furthermore, $P$. yoelii spect2(-) parasite-infected cells did not show substantial levels of apoptosis, even after $24 \mathrm{~h}$ when wild-type parasite-infected cells undergo significant apoptosis (Figure 2c). Because p52(-)p36(-)parasites arrest soon after infection, and infected cells do not survive, ${ }^{10}$ we could not monitor apoptosis in p52(-)p36(-) parasite-infected hepatocytes later during infection.

If wild-type parasite-infected hepatocytes remain unwounded, parasite entry must have occurred during the formation of a PVM (Figure 2d). Therefore, we marked wounded cells by including fluorescently labeled dextran in the hepatocyte culture media before parasite addition. As before, we marked the presence of intracellular parasites using an antibody against CSP. After $24 \mathrm{~h}$, we observed that parasite-harboring host cells that had been wounded exhibited higher levels of apoptosis than parasite-harboring hepatocyte that were not wounded (Figure 2e). This suggests that wild-type parasites that enter a hepatocyte without PVM formation are more likely to induce apoptosis in their host cell than parasites that invade with forming a PVM. The rate of apoptosis is higher in wounded cells containing parasites than in wounded cells without parasites, suggesting that cell wounding itself does not initiate substantial levels of apoptosis. Rather, the presence of the LS parasite not ensconced by a PVM results in host cell death. Taken together, these observations indicate that the LS PVM has an important role in protecting the infected host hepatocyte from apoptosis.

LS-infected host cells partially resist Fas-mediated apoptosis. Fas ligand and its receptor Fas (CD95) constitute a canonical pathway of cytotoxic T-lymphocyte- and natural killer cell-driven apoptosis in the liver, ${ }^{15-17}$ suggesting that the LS parasite has encountered this type of proapoptotic stimulus throughout its evolutionary history. We therefore asked if LS-infected cells are susceptible to Fas activation after infection (Figure 3a). We found that uninfected cells undergo significant levels of apoptosis in response to treatment with the Jo-2 Fas-activating antibody (Figure 3b), whereas LS-infected cells do not exhibit substantially elevated levels of apoptosis in response to the same treatment (Figure 3c). These data demonstrate that a population of $P$. yoelii-infected host cells has the capacity to effectively resist Fas-mediated apoptosis. However, the ability of infected cells to resist Fas-mediated apoptosis is not absolute, as Jo-2-treated, infected cultures showed somewhat lower total numbers of LSs than untreated controls (Figure 3d). This further supports the hypothesis that not all LS-infected cells are able to effectively block apoptosis. Consequently, LS-infected cells can be categorized into three populations: (i) infected host cells that undergo apoptosis without additional external stimuli, (ii) infected host cells that resist apoptosis even in the presence of potent external stimuli such as Fas activation, and (iii) infected host cells whose apoptotic state can be tipped from survival to death with external stimuli.

Mitochondrial signaling has a critical role in regulating host hepatocyte apoptosis during LS infection. Hepatocytes are 'type II cells' and thus preferentially utilize mitochondrial signaling to facilitate both intrinsic and extrinsic apoptosis. Mitochondrial control of apoptosis is tightly regulated by a family of $\mathrm{BH} 3-$ domain-containing proteins, which participate in direct protein-protein interactions to provide cellular resistance or sensitivity to apoptosis (Figure $4 \mathrm{a}$ ). ${ }^{18}$ Cleavage of Bid (to produce t-Bid) is required for initiation of mitochondrial apoptosis; therefore, elimination of Bid generally inhibits intrinsic and extrinsic apoptosis in hepatocytes. To determine if the mitochondrial pathway is responsible for mediating apoptosis in the population of LSinfected cells that cannot survive under basal conditions, we compared total $P$. yoelii LS burden in wild-type and Bid $(-/-)$ mice. We found that $\mathrm{Bid}(-/-)$ mice had substantially elevated LS parasite burden (Figure $4 b$ ) similar to the increase observed when inhibiting all apoptosis with Q-VD-OPh pancaspase inhibitor treatment (Figure 2c). The data show that the mitochondrial apoptotic cascade has a significant role in eliminating LS-infected hepatocytes in vivo. Thus, mitochondrial apoptosis is a key node of susceptibility for LS -infected hepatocytes. Consequently, infected hepatocytes could be hypersusceptible to the activation of the mitochondrial apoptotic pathway.

Apoptotic susceptibility of LS-infected hepatocytes is enhanced by targeting key mitochondrial signaling nodes. B-cell lymphoma 2 (Bcl-2) family members are critical for blocking pro-apoptotic signaling in the mitochondria, and inhibition of these proteins leads to mitochondriadriven apoptosis. We utilized the well-characterized $\mathrm{Bcl}-2$ family inhibitor, $A B T-737,{ }^{19}$ and found that increasing concentrations of ABT-737 ranging from 50 to $200 \mathrm{nM}$ (Figure 5a) increased levels of apoptosis in uninfected Hepa 1-6 cells. To exclude any direct effects on the parasite, we tested ABT-737 on Plasmodium falciparum blood stages, and found that it had no substantial effect at even the highest concentration used (Figure 5b). We then asked if boosting pro-apoptotic signaling in the host cell mitochondria using ABT-737 had a substantial impact on LS-infected host cell survival. Indeed, LS burden was significantly decreased when infected Hepa 1-6 cultures were treated with ABT-737 ranging in concentration from 50 to $200 \mathrm{nM}$ (Figure $5 \mathrm{c}$ ). Importantly, ABT-737 was able to significantly reduce LS parasite burden with doses much lower than those used to treat cancer cells. ${ }^{19,20}$ Strikingly, we found that at lower concentrations (50 and $75 \mathrm{nM}$ ) LS-infected cells were heavily 
a

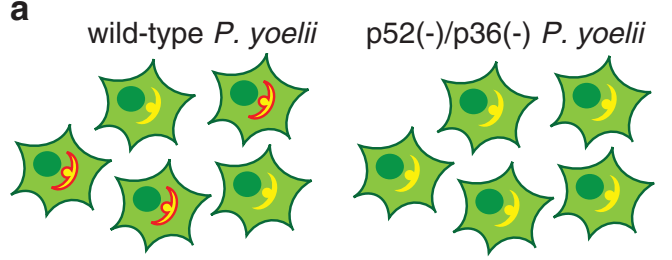

b

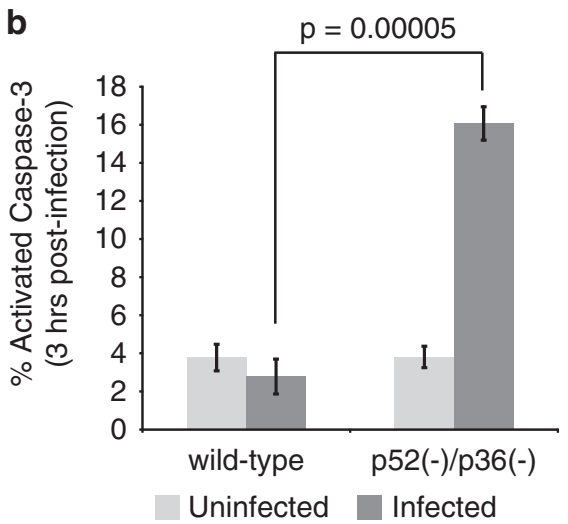

d

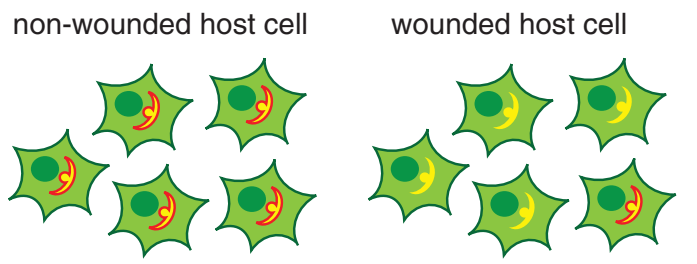

Spect2(-) P. yoelii

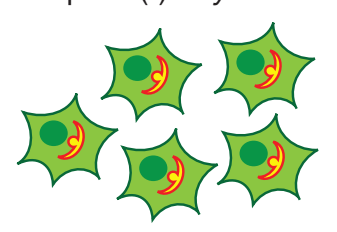

C
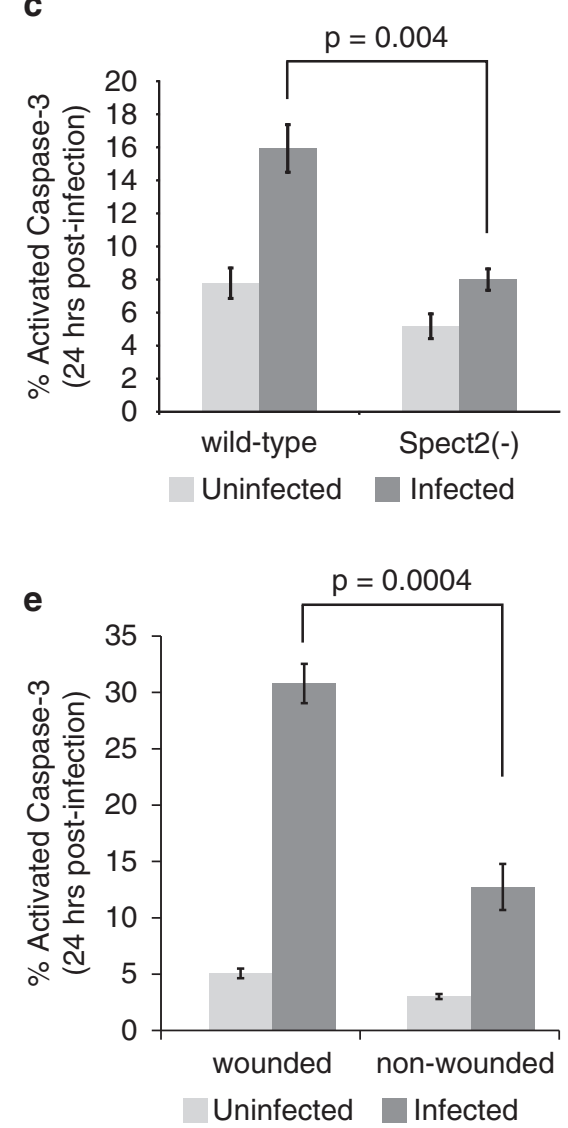

Figure 2 The PVM is critical for maintaining parasite control over apoptosis in infected hepatocytes. (a) Cartoons depict defects in each knockout parasite: wild-type parasites can enter hepatocytes with or without a vacuole, whereas p52(-)/p36(-) parasites cannot form a vacuole. Spect2( - ) parasites cannot traverse and thus can only invade hepatocytes by establishing a PVM. (b and $\mathbf{c}$ ) Percentage of hepatocytes positive for cleaved caspase-3 was evaluated in infected and uninfected populations. (b) p52( - )/p36( - )-infected cells elicit a strong apoptotic response $3 \mathrm{~h}$ after infection. (c) Spect2 ( ) transgenic parasites do not elicit an apoptotic response after $24 \mathrm{~h}$ of infection. (d) Cartoons depict the role of cell wounding and PVM formation. Parasites that enter a cell by wounding (traversal) do so without the formation of a PVM. Thus, the population of host cells that are wounded and contain a parasite is heavily enriched for parasites without a PVM. Parasites that reside within a host cell that has not been wounded have invaded with the formation of a PVM. (e) Parasite-containing host cells exhibit higher levels of apoptosis when parasites have entered by traversal as opposed to when parasites have invaded with PVM formation. Error bars depict S.D. of biological replicates

sensitized to ABT-737, exhibiting far higher levels of apoptosis than uninfected cells (Figure $5 \mathrm{~d}$ ). Thus, enabling mitochondrial apoptosis is effective at preferentially eliminating infected hepatocytes. Interestingly, Spect2(-) LSinfected hepatocytes, as well as wild-type-infected cells with or without an intact PVM, all exhibited this sensitivity, suggesting that Bcl-2 family inhibition overwrites any antiapoptotic signals initiated by LS parasites (Supplementary Figure 3).

We next investigated if another Bcl-2 family inhibitor could prove as effective at clearing parasite-infected hepatocytes while having little impact on uninfected hepatocytes. We chose to utilize obatoclax mesylate, a pan-Bcl-2 family inhibitor with low levels of toxicity, which is orally bioavailable. ${ }^{21,22}$ We found that obatoclax induces a measurable but substantially lower level of apoptosis in uninfected Hepa1-6 cells when compared with ABT-737 (Figure 5e), and, like ABT-737, it did not directly kill blood stage parasites at the concentrations used (Figure 5f). Yet, obatoclax reduced the number of LS-infected cells in vitro by $>70 \%$ (Figure $5 \mathrm{~g}$ ), but at the same concentrations it had limited effects on apoptosis in uninfected cells (Figure 5h). 
a

Infect Hepa1-6 cells with $P$. yoeli parasites

b

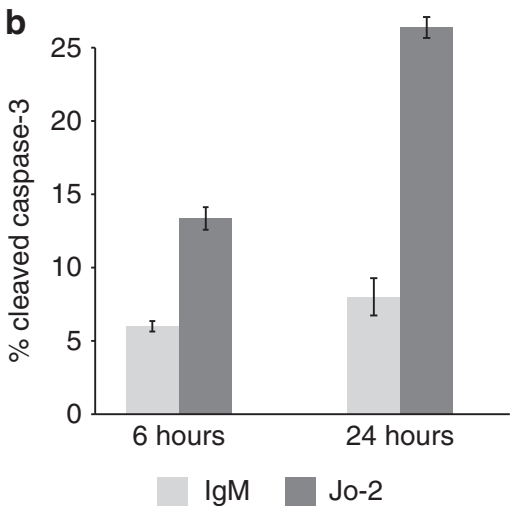

Induce apoptosis with Jo-2 antibody

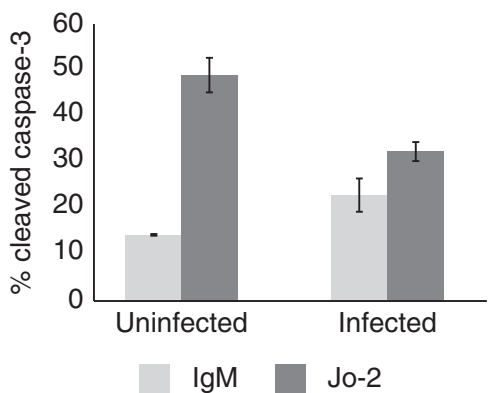

\section{C}

Detach, fix and stain cells with $\alpha$ CS-Alexafluor488, $\alpha$ Cleaved Caspase-3-PE d

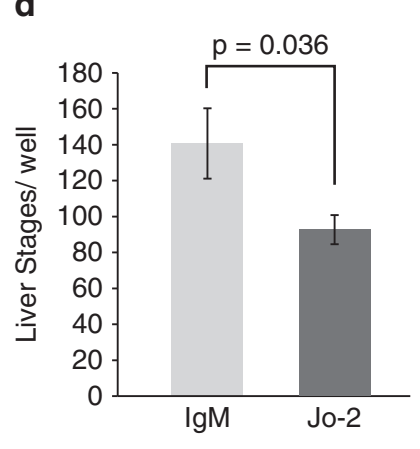

Figure 3 Infected hepatocytes are partially resistant to Fas-mediated apoptosis. (a) Schematic representation of methodology used to monitor $P$. yoelii-infected hepatocytes and caspase-3 cleavage using flow cytometry. (b) $\alpha$ Fas antibody Jo-2 induces significant apoptosis in Hepa1- 6 cells. (c) Cells were treated with $\alpha$ Fas antibody for $24 \mathrm{~h}$, beginning $90 \mathrm{~min}$ after infection. Infected cells are less susceptible to Fas-mediated apoptosis than uninfected cells. (d) $\alpha$ Fas antibody eliminates some LS-infected cells. Hepa 1-6 cells were treated with $\alpha$ Fas antibody for $24 \mathrm{~h}$, beginning $90 \mathrm{~min}$ after infection. Total parasites were quantified by microscopy and manual counting

a

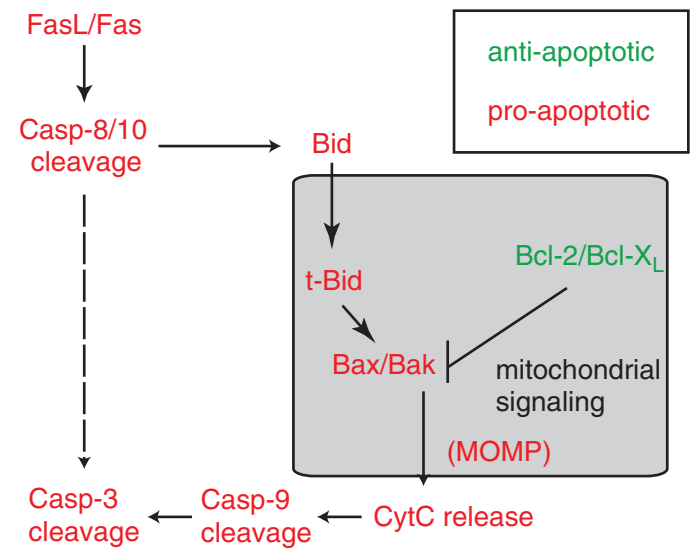

b

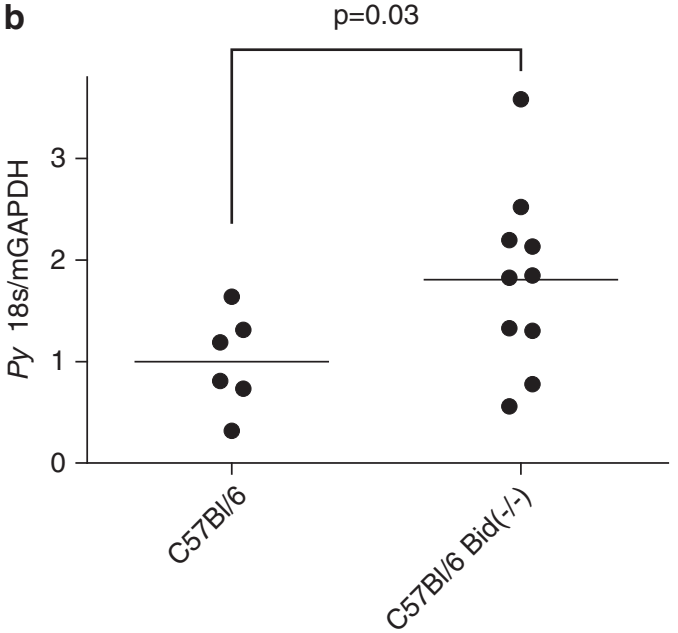

Figure 4 Mitochondria-driven apoptosis eliminates LS-infected hepatocytes in vivo. (a) Schematic of apoptosis in hepatocytes. Apoptosis can be initiated either through the mitochondria or through the more direct activity of caspase 8/10. The mitochondrial pathway is preferred in most hepatocytes. (b) Bid knockout mice have increased susceptibility to $P$. yoelii LS infection. Bid knockout or wild-type C57B//6 mice were infected with 100000 P. yoelii sporozoites. LS burden was monitored $42 \mathrm{~h}$ after infection by quantitative PCR

Finally, to extend the findings to an animal model, we treated BALB/cJ mice with $5 \mathrm{mg} / \mathrm{kg}$ obatoclax or vehicle alone (9.6\% polyethylene glycol $300,0.4 \%$ polysorbate 20 , and $5 \%$ dextrose), infected the mice with 100000 P. yoelii sporozoites, and measured LS burden after $42 \mathrm{~h}$. Infected mice treated with obatoclax had dramatically lower LS parasite burden when compared with vehicle-treated infected mice (Figure 5i). Obatoclax treatment was well tolerated, and mice appeared healthy after $72 \mathrm{~h}$ of treatment. The data demonstrate that sensitizing LS-infected hepatocytes to apoptosis can substantially reduce parasite burden in the liver.

\section{Discussion}

Interactions between the malaria parasite LS and its hepatocyte host are complex from the time of sporozoite invasion until the ultimate release of first-generation red blood cell-infectious forms. ${ }^{23}$ The parasite has evolved to control and exploit its host cell, thereby ensuring successful transition to blood infection and transmission. The PVM constitutes the principal host-parasite interface and is critical to mediate this exploitative relationship. We demonstrate that the ability of the LS to form a PVM can alter the balance between survival and apoptosis of the infected hepatocyte. It has been previously 
shown by van Dijk et al. ${ }^{10}$ that $P$. berghei $p 52(-)$ parasites induce apoptosis in their host hepatocyte soon after infection. It remains unclear if these parasites are able to initially form or partially form a PVM during invasion, and thus it remains to be seen if parasites, which die within their host hepatocyte, also induce apoptosis if a PVM is formed before developmental arrest. It also remains unknown whether loss of PVM integrity simply exposes the hepatocyte to pro-apoptotic attributes of
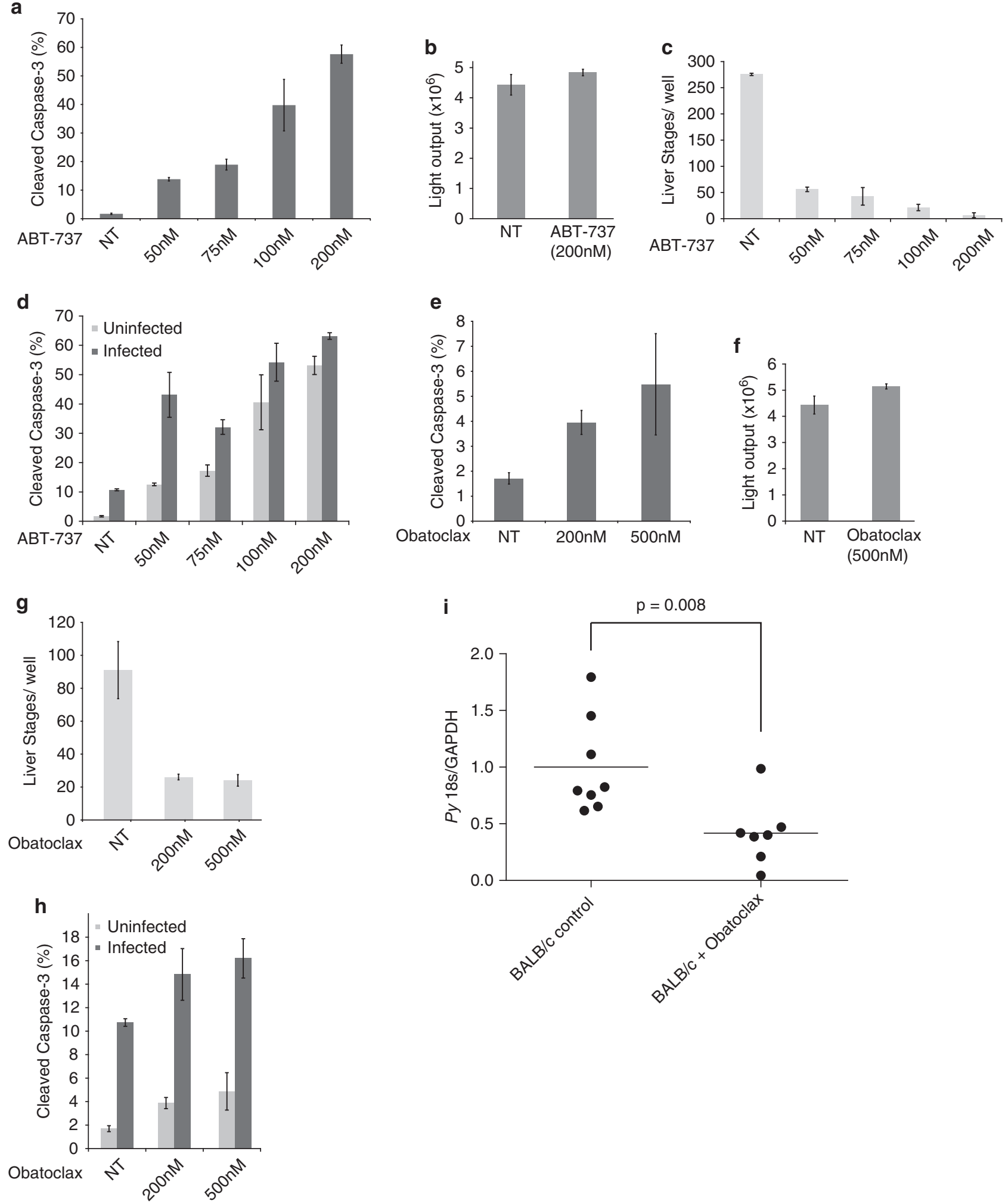
the parasite or if aspects of the PVM are actively engaged in modulating hepatocyte apoptosis.

The premature death of the infected hepatocyte not only ends the life of the LS contained within but also affects the surrounding liver tissue and generates immune responses that can have an impact on subsequent infections. A prosurvival response in the infected cell allows the parasite to thrive and remain immunologically inert. Although it is tempting to characterize the signaling in the infected hepatocyte as strictly pro-survival or pro-death, the data presented here suggest a more complex dynamic relationship between the LS parasite and its host hepatocyte than previously appreciated. The interaction between a subset of parasites and their host hepatocyte results in infected host cell death; however, the majority of parasites in the population impose survival of the infected hepatocytes, and these also actively resist Fas-mediated apoptosis. Interestingly, LS-infected hepatocytes are unable to resist mitochondrialdriven apoptotic stimuli. Thus, mitochondrial signaling in the infected hepatocyte represents a key node of susceptibility for LS parasite infection.

It has been previously demonstrated that $P$. berghei LSinfected hepatoma cells can resist apoptosis that is initiated by cycloheximide and tumor necrosis factor ${ }^{5}$ in combination, or t-butyl-hydroperoxide. ${ }^{4}$ These stimuli that are not physiologically relevant or present in the resting liver. It has also been shown that infected host cells undergo non-apoptotic cell death at the end of LS development, presumably to foster release of infectious forms into the blood stream. ${ }^{24}$ Here we demonstrate for the first time that infected hepatocytes respond differently to diverse apoptotic stimuli. These findings highlight the importance of analyzing LS-infected cells not strictly on a population level, but also addressing the variation within the population. Moving forward, single-cell analyses will be critical to a more comprehensive understanding of the intricate interplay of LS parasites and their hepatocyte hosts.

The complex perturbations caused by Plasmodium LS infection are multifaceted stimuli for the infected hepatocyte. Although it is tempting to simplify the hepatocyte response to LS infection as an additive response to the collection of stimuli present, work in the last decade has demonstrated that combinations of cellular stimuli do not always behave as a simple sum of their parts. ${ }^{25}$ For instance, cells that proliferate in response to growth factor, or undergo low-level apoptosis in response to tumor necrosis factor, undergo substantially elevated levels of apoptosis when treated with growth factor and tumor necrosis factor in combination. ${ }^{26}$ In cancer treatment, the role of mitochondrial apoptotic signaling in combination with other cellular events is already being translated to the clinic; drugs that target the $\mathrm{Bcl}-2$ pathways are being tested as sensitization agents in combination with more traditional chemotherapy. ${ }^{27}$ Additional insight regarding the wiring of signaling networks in hepatocytes will provide a framework to more fully understand the nuances of the interaction between the host hepatocyte and LS parasite, and also additional insights into the optimal points of intervention.

Although there is no evidence that LS parasites transform their host cell, recent evidence suggests that $P$. yoelii-infected hepatocytes are not entirely distinct from cancer cells. We have demonstrated that averaged over the population, LSinfected hepatocytes exhibited elevated levels of proteins regulating cell cycle progression and survival, and diminished levels of proteins involved in autophagic and apoptotic signaling. ${ }^{28}$ Here we demonstrate that although many parasites successfully evade hepatocyte defenses and protect their host cell against extrinsic apoptosis, a subset of LS parasites engage the apoptotic machinery and are cleared from the liver, along with their host hepatocytes. In addition, our previous work demonstrated that mitochondrial proteins $\mathrm{Bcl}-2$ and Bad were skewed toward anti-apoptotic signaling; ${ }^{28}$ here we show that reversing this perturbation by inhibiting the $\mathrm{Bcl}-2$ family overcomes the apoptotic block placed by the parasite and thus eliminates infected cells. Interestingly, the parasite-driven modulation of another hepatocyte protein, p53, exhibits a similar pattern: the parasite actively suppresses hepatocyte $\mathrm{p} 53$, but when this suppression is reversed with a small molecule, infected cells are eliminated. ${ }^{28}$ As additional data are generated that fully characterize the host cell-signaling milieu in which the LS parasite thrives, a more comprehensive understanding of the points of susceptibility will emerge. It is enticing to ask if combinations of host-targeted drugs, aimed at both activating p53 and mitochondrial apoptosis, could lead to the complete elimination of LS infected cells, and thus the onset of clinical malaria.

The efficacy of host-based therapeutics aimed at the mitochondrial apoptotic cascade might be restricted to hepatotropic pathogens, or might even be exclusive to Plasmodium species. The hepatocyte microenvironment that houses the malaria LS parasite is unique in many ways and presents a finite set of challenges for the developing LS to overcome. LS parasites can resist Fas stimulation but cannot resist apoptotic insults initiated in the mitochondria. By comparison, Toxoplasma species, unlike Plasmodium, invade a variety of cell types, and thus come in contact with

Figure 5 Bcl-2 family-targeted drugs eliminate LS-infected hepatocytes in vitro and in vivo via apoptosis. (a) ABT-737 induces apoptosis in Hepa 1-6 cells in a doseresponse manner. Cells were treated with the indicated concentration of ABT-737 for $24 \mathrm{~h}$. (b) Transgenic $P$. falciparum parasites that express a GFP-luciferase fusion were used to monitor growth in asexual blood stage culture. There is no difference between the signal obtained from drug-treated and untreated parasites. Parasites were synchronized as ring stages and allowed to develop for $48 \mathrm{~h}$. Drug was replaced daily. (c) ABT-737 robustly eliminates P. yoelii parasite-infected host cells in vitro. LSs were visualized by microscopy and quantified by manual counting. Cells were treated with the indicated concentration of ABT-737 for $24 \mathrm{~h}$, beginning $90 \mathrm{~min}$ after infection. (d) LS-infected host cells are more susceptible to ABT-737-mediated apoptosis than uninfected cells. Cells were treated with indicated concentrations of ABT-737 for $24 \mathrm{~h}$, beginning $90 \mathrm{~min}$ after infection. Populations of caspase-3-positive cells were examined in infected and uninfected populations by flow cytometry. (e) Obatoclax induces apoptosis in Hepa 1-6 cells. Cells were treated with indicated concentrations of obatoclax for $24 \mathrm{~h}$. (f) Transgenic $P$. falciparum parasites that express GFP-luciferase fusion were used to monitor growth in asexual blood stage culture. There is no significant difference between the signal obtained from drug-treated and untreated parasites. (g) Obatoclax eliminates $P$. yoelii LS-infected host cells in vitro. LSs were visualized by microscopy and quantified by manual counting. (h) Infected cells are more susceptible to obatoclaxmediated apoptosis than uninfected cells. Cells were treated with indicated concentrations of obatoclax for $24 \mathrm{~h}$, beginning $90 \mathrm{~min}$ after infection. Populations of caspase-3positive cells were examined in infected and uninfected populations by flow cytometry. (i) Obatoclax effectively eliminates LS-infected hepatocytes in vivo. BALB/cJ mice were treated with $5 \mathrm{mg} / \mathrm{kg}$ obatoclax once daily and infected with 100000 P. yoelii sporozoites. LS burden was monitored by quantitative PCR $42-44 \mathrm{~h}$ after infection 
a greater diversity of challenges. It has been demonstrated that many components of the mitochondrial apoptotic signaling network are strongly inhibited in Toxoplasmainfected cells, ${ }^{29-32}$ suggesting that these parasites, unlike Plasmodium, might be able to resist even mitochondrialtargeted insults. ${ }^{32}$ It remains an unanswered question if more host cell-promiscuous parasite species have the capacity to resist a wider variety of apoptotic stimuli.

Parasite evolution has been a challenge for combating malaria, as rapidly replicating parasites frequently become drug resistant. ${ }^{2}$ Targeting critical host signaling node(s) could provide protection against the development of drug resistance. However, to date, little attention has been given to develop host-based prophylaxis aimed at selectively eliciting apoptosis in infected cells, perhaps because of early studies demonstrating that infected cells could resist apoptosis. ${ }^{4,5}$ Here we provide evidence that initiating apoptosis by targeting the mitochondrial cascade preferentially targets Plasmodiuminfected hepatocytes. These findings argue for further investigation into, and broadening the targets of, host-based strategies to prevent malaria.

\section{Materials and methods \\ Cell lines and culture. Hepa 1-6 Cells were a gift from Ana Rodriguez (NYU). Cells were maintained in Dulbecco's Modified Eagle Medium (DMEM) complete media (Cellgro, Manassas, VA, USA), supplemented with 10\% FBS (Sigma-Aldrich, St. Louis, MO, USA), $100 \mathrm{IU} / \mathrm{ml}$ penicillin (Cellgro), $100 \mu \mathrm{g} / \mathrm{ml}$ streptomycin (Cellgro), $2.5 \mu \mathrm{g} / \mathrm{ml}$ fungizone (HyClone/Thermo Fisher, Waltham, MA, USA), and $5 \mu \mathrm{g} / \mathrm{ml}$ gentamicin (BioWhittaker/Lonza, Basel, Switzerland), and split 1-2 times weekly. Where indicated, cells were treated with ABT-737 (SelleckChem, Houston, TX, USA) and obatoclax mesylate (SelleckChem) at indicated concentrations. Both molecules were dissolved in DMSO for cell culture experiments.}

Mosquito rearing and sporozoite production. For $P$. yoelii sporozoite production, female 6-8-week-old Swiss Webster mice (Harlan, Indianapolis, IN, USA) were injected with blood stage $P$. yoelii (17XNL) parasites to begin the growth cycle. Animal handling was conducted according to the Institutional Animal Care and Use Committee-approved protocols. We used infected mice to feed female Anopheles stephensi mosquitoes after gametocyte exflagellation was observed. We isolated salivary gland sporozoites according to the standard procedures at days 14 or 15 post blood meal. For each experiment, salivary glands were isolated in parallel in order to ensure that sporozoites were extracted from salivary glands under the same conditions.

Quantification of liver burden by real-time RT-PCR. Total RNA was extracted using TRIzol reagent (Invitrogen, Grand Island, NY, USA). CDNA synthesis was performed using the Super Script III Platinum two-step qRT-PCR kit according to the manufacturer's instructions (Invitrogen). All PCR amplification cycles were performed at $95^{\circ} \mathrm{C}$ for $30 \mathrm{~s}$ for DNA denaturation, and $60^{\circ} \mathrm{C}$ for 4 min for primer annealing and DNA-strand extension. Parasite $18 \mathrm{~S}$ was amplified using primers with sequences: $5^{\prime}$-GGGGATTGGTTTTGACGTTTTTGCG-3' and $5^{\prime}$ AAGCATTAAATAAAGCGAATACATCCTTAT-3'. Mouse GAPDH was amplified using sequences $5^{\prime}$-CCTCAACTACATGGTTTACAT-3' and 5'-GCTCCTGGAA GATGGTGATG- $3^{\prime}$. For quantitative PCR, a standard curve was generated using $1: 4$ dilutions of a reference cDNA sample for PCR amplification of all target PCR products. Experimental samples were compared with this standard curve to give a relative abundance of transcript.

Immunofluorescence. In all, $1.5 \times 10^{5}$ Hepa 1-6 cells were grown in DMEM complete medium in each well of an eight-well Permanox slide (VWR, Radnor, PA, USA). Cells were infected with $5 \times 10^{4} P$. yoelii sporozoites. Slides were centrifuged for $3 \mathrm{~min}$ at $515 \mathrm{~g}$ in a hanging-bucket centrifuge to aid in sporozoite invasion. After $90 \mathrm{~min}$, we removed media that contained sporozoites that had not infected the cells and added fresh media. We allowed LS parasites to develop for $24 \mathrm{~h}$, at which time cells were fixed with $4 \%$ paraformaldehyde, blocked, and permeabilized for $1 \mathrm{~h}$ in PBS with the addition of $0.1 \%$ Triton X-100 and $2 \%$ bovine serum albumin (BSA). Staining steps were performed in PBS supplemented with $2 \%$ BSA. We stained cells using anti-sera to Plasmodium heat shock protein 70 (HSP70) and upregulated in infectious sporozoites 4 protein (UIS4) $4{ }^{\circ} \mathrm{C}$ overnight and then washed several times, and antibodies were visualized with the use of AlexaFluor-488 goat anti-mouse and AlexaFluor-594 goat anti-rabbit secondary antibody (Invitrogen). We used DAPI stain to visualize both hepatocyte and parasite nuclei. Sporozoites that had not invaded and/or developed in hepatoma cells were distinguished by UIS4 non-circumferential staining and morphology.

Quantification of LS parasites after $\alpha$ Fas, ABT-737, or obatoclax treatment by manual counting. In all, $1.5 \times 10^{5}$ Hepa 1-6 cells were grown in DMEM complete medium in each well of an eight-well Permanox slide. For $\alpha$ Fas experiments, cells were treated with Jo-2 (BD Biosciences, San Jose, CA, USA) or IgM control for $24 \mathrm{~h}$. For ABT-737 and obatoclax experiments, cells were treated with DMSO alone or drug for $24 \mathrm{~h}$ as described above. Cells were infected with $5 \times 10^{4} \mathrm{P}$. yoelii sporozoites. Slides were centrifuged for $3 \mathrm{~min}$ at $515 \mathrm{~g}$ in a hanging-bucket centrifuge to aid in sporozoite invasion. After $90 \mathrm{~min}$, we removed media that contained sporozoites that had not infected the cells and added fresh media. We allowed LSs to develop for $24 \mathrm{~h}$, at which time cells were fixed with $4 \%$ paraformaldehyde, blocked, and permeabilized for $1 \mathrm{~h}$ in PBS with the addition of $0.1 \%$ Triton X-100 and $2 \%$ BSA. Staining steps were performed in PBS supplemented with $2 \%$ BSA. We stained cells using anti-sera to Plasmodium HSP70 and UIS4 proteins at $4{ }^{\circ} \mathrm{C}$ overnight and then washed several times, and antibodies were visualized with the use of AlexaFluor-488 goat anti-mouse and AlexaFluor-594 goat anti-rabbit secondary antibody (Invitrogen). We used DAPI stain to visualize both hepatocyte and parasite nuclei. Sporozoites that had not invaded and/or developed in hepatoma cells were distinguished by UIS4 noncircumferential staining and morphology. All LSs in each well were counted, and each assay was performed in biological triplicate.

Quantification of LS parasites by FACS. Cells were cultured as described above. In all, $3 \times 10^{5}$ or $6 \times 10^{5}$ cells were plated in each well of a $24-$ well or 12-well plate and infected with $10^{5}$ or $2 \times 10^{5} P$. yoelii sporozoites, respectively. Cells were treated with or without ABT-737 or obatoclax, as described above. At the desired time point, cells were trypsinized and then fixed with Perm/Fix buffer (BD Biosciences). Cells were blocked in Perm/Wash buffer (BD Biosciences) supplemented with $2 \%$ BSA. Additional staining steps were performed in Perm/Wash buffer alone. We stained cells using the monoclonal antibody to CSP conjugated to AlexaFluor-488 or AlexaFluor-647, as well as an antibody to cleaved caspase-3 conjugated to phycoerythrin (PE; Cell Signaling Technology, Danvers, MA, USA) at $25^{\circ} \mathrm{C}$. Parasitized and apoptotic hepatocytes were identified by FACS. Analysis was performed on BD LSRII. Flow-cytometry analysis was performed using FlowJo software (TreeStar). All experimental conditions were tested in biological triplicate. All data are representative of three independent experiments.

Quantification of LS, traversal and apoptosis by FACS. Cells were cultured as described above. Cells $\left(1.2 \times 10^{6}\right)$ were plated in each well of a sixwell plate and infected with $4 \times 10^{6} \mathrm{P}$. yoelii sporozoites. A concentration of $400 \mu \mathrm{g} / \mathrm{ml} 10000 \mathrm{MW}$ dextran conjugated to Alexa Fluor 488 (Invitrogen) was added at the time of infection to assess cell permeabilization due to sporozoite traversal. At the desired time point, cells were trypsinized and then fixed with Perm/Fix buffer (BD Biosciences). Cells were blocked in Perm/Wash buffer (BD Biosciences) supplemented with $2 \%$ BSA. Additional staining steps were performed in Perm/Wash buffer alone. We stained cells using the monoclonal antibody to CSP conjugated to Alexa Fluor 647, as well as an antibody to cleaved caspase-3 conjugated to PE (Cell Signaling Technology) at $25^{\circ} \mathrm{C}$. Parasitized, traversed, and apoptotic hepatocytes were identified by FACS. Analysis was performed on BD LSRII. Flow-cytometry analysis was performed using FlowJo software (TreeStar, Ashland, OR, USA). All experimental conditions were tested in biological triplicate. All data are representative of three independent experiments.

$\operatorname{Bid}\left(-I_{-}\right)$in vivo experiments. Mice bred from heterozygous B6.129 $\times 1$-Bidtm1Sjk/J mice were genotyped as follows. DNA was extracted from each mouse with the Gentra Puregene Kit A (Qiagen, Valencia, CA, USA) using the protocol for DNA purification from mouse tail tissue. PCR amplification of 
the purified DNA from each mouse was amplified according to the Jax genotyping specifications; the common reverse primer (olMR9558; 5'-CCGAAATGTCCCATAAGAG-3'), wild-type forward primer (olMR9559; 5'-GAGATGGACCACAACATC-3'), and mutant forward primer (olMR9560; 5'-TGCTACTTCCATTT GTCACGTCCT-3') were used to identify the wild-type (124bp) and PGK-neo cassette $(350 \mathrm{bp})$ products.

In vivo obatoclax experiments. Eighteen BALB/CJ mice (Jackson Laboratory, Bar Harbor, ME, USA) were treated with either vehicle control or $5 \mathrm{mg} / \mathrm{kg}$ of obatoclax by oral gavage once daily for 3 days. On the second day of treatment, mice were injected with $10^{5} P$. yoelii sporozoites. Livers were excised from mice at $44 \mathrm{~h}$ after infection. Animal handling was conducted according to the Institutional Animal Care and Use Committee-approved protocols.

In vivo Q-VD-OPh experiments. Thirty BALB/cJ mice (Jackson) were treated with either vehicle control or $20 \mathrm{mg} / \mathrm{kg}$ of Q-VD-OPh (SM Biochemicals LLC, Anaheim, CA, USA) once daily for 3 days. On the second day of treatment, mice were injected with $10^{5} \mathrm{P}$. yoelii sporozoites. Livers were excised from mice at $44 \mathrm{~h}$ after infection. Animal handling was conducted according to the Institutional Animal Care and Use Committee-approved protocols.

\section{Conflict of Interest}

The authors declare no conflict of interest.

Acknowledgements. We are grateful to William W. Betz and Jen C.C. Hume for mosquito and sporozoite production. We thank Hieu Nguyen for technical assistance with FACS and Marian Abdullahi for excellent technical assistance with in vitro experiments. We thank Roberto Guirnalda, Lynett Robertson and Seattle Biomedical Research Institute vivarium staff for their work with mice. AK is a recipient of a NRSA Ruth L. Kirschstein National Research Service Award (F32 Al091129), which has partially funded this work. This work has also been partially funded by 1R01GM101183-01A1 to SHIK.

\section{Author contributions}

AK and SHIK designed the research. AK, PGM, AND, SAM, VL, and HSK performed the experiments. AK, PGM, AND and SHIK analyzed the data. AK and SHIK wrote the paper with input from all others.

1. Mackinnon MJ, Marsh K. The selection landscape of malaria parasites. Science 2010; 328 866-871.

2. Fidock DA. Drug discovery: priming the antimalarial pipeline. Nature 2010; 465: 297-298.

3. Kappe SH, Vaughan AM, Boddey JA, Cowman AF. That was then but this is now: malaria research in the time of an eradication agenda. Science 2010; 328: 862-866.

4. van de Sand C, Horstmann S, Schmidt A, Sturm A, Bolte S, Krueger A et al. The liver stage of Plasmodium berghei inhibits host cell apoptosis. Mol Microbiol 2005; 58: 731-742.

5. Leiriao P, Albuquerque SS, Corso S, van Gemert GJ, Sauerwein RW, Rodriguez A et al. HGF/MET signaling protects Plasmodium-infected host cells from apoptosis. Cell Microbio 2005; 7: 603-609.

6. Yin XM, Wang K, Gross A, Zhao Y, Zinkel S, Klocke B et al. Bid-deficient mice are resistant to Fas-induced hepatocellular apoptosis. Nature 1999; 400: 886-891.

7. Wei MC, Zong WX, Cheng EH, Lindsten T, Panoutsakopoulou V, Ross AJ et al Proapoptotic $B A X$ and BAK: a requisite gateway to mitochondrial dysfunction and death Science 2001; 292: 727-730.

8. Kaushansky A, Rezakhani N, Mann H, Kappe SH. Development of a quantitative flow cytometry-based assay to assess infection by Plasmodium falciparum sporozoites. Mol Biochem Parasitol 2012; 183: 100-103.

9. Prudencio M, Mota MM, Mendes AM. A toolbox to study liver stage malaria. Trends Parasitol 2011; 27: 565-574.

10. van Dijk MR, Douradinha B, Franke-Fayard B, Heussler V, van Dooren MW, van Schaijk B et al. Genetically attenuated, P36p-deficient malarial sporozoites induce protective immunity and apoptosis of infected liver cells. Proc Natl Acad Sci USA 2005; 102 12194-12199.
11. Mueller AK, Camargo N, Kaiser K, Andorfer C, Frevert U, Matuschewski $\mathrm{K}$ et al. Plasmodium liver stage developmental arrest by depletion of a protein at the parasite-host interface. Proc Natl Acad Sci USA 2005; 102: 3022-3027.

12. Mikolajczak SA, Jacobs-Lorena V, MacKellar DC, Camargo N, Kappe SH. L-FABP is a critical host factor for successful malaria liver stage development. Int J Parasitol 2007; 37: 483-489.

13. Mota MM, Pradel G, Vanderberg JP, Hafalla JC, Frevert U, Nussenzweig RS et al. Migration of Plasmodium sporozoites through cells before infection. Science 2001; 291: 141-144.

14. Labaied M, Harupa A, Dumpit RF, Coppens I, Mikolajczak SA, Kappe SH. Plasmodium yoelii sporozoites with simultaneous deletion of P52 and P36 are completely attenuated and confer sterile immunity against infection. Infect Immun 2007; 75: 3758-3768.

15. Chirmule N, Moscioni AD, Qian Y, Qian R, Chen Y, Wilson JM. Fas-Fas ligand interactions play a major role in effector functions of cytotoxic $T$ lymphocytes after adenovirus vectormediated gene transfer. Hum Gene Ther 1999; 10: 259-269.

16. Strasser A, Jost PJ, Nagata $S$. The many roles of FAS receptor signaling in the immune system. Immunity 2009; 30: 180-192.

17. Smyth MJ, Cretney E, Kelly JM, Westwood JA, Street SE, Yagita H et al. Activation of NK cell cytotoxicity. Mol Immunol 2005; 42: 501-510.

18. Happo L, Strasser A, Cory S. BH3-only proteins in apoptosis at a glance. J Cell Sci 2012; 125: 1081-1087.

19. van Delft MF, Wei AH, Mason KD, Vandenberg CJ, Chen L, Czabotar PE et al. The BH3 mimetic ABT-737 targets selective Bcl-2 proteins and efficiently induces apoptosis via Bak/ Bax if Mcl-1 is neutralized. Cancer Cell 2006; 10: 389-399.

20. Kline MP, Rajkumar SV, Timm MM, Kimlinger TK, Haug JL, Lust JA et al. ABT-737, an inhibitor of Bcl-2 family proteins, is a potent inducer of apoptosis in multiple myeloma cells. Leukemia 2007; 21: 1549-1560.

21. Trudel S, Li ZH, Rauw J, Tiedemann RE, Wen XY, Stewart AK. Preclinical studies of the pan-Bcl inhibitor obatoclax (GX015-070) in multiple myeloma. Blood 2007; 109: 5430-5438.

22. O'Brien SM, Claxton DF, Crump M, Faderl S, Kipps T, Keating MJ et al. Phase I study of obatoclax mesylate (GX15-070), a small molecule pan-Bcl-2 family antagonist, in patients with advanced chronic lymphocytic leukemia. Blood 2009; 113: 299-305.

23. Vaughan AM, Aly AS, Kappe SH. Malaria parasite pre-erythrocytic stage infection: gliding and hiding. Cell Host Microbe 2008; 4: 209-218.

24. Sturm A, Amino R, van de Sand C, Regen T, Retzlaff S, Rennenberg A et al. Manipulation of host hepatocytes by the malaria parasite for delivery into liver sinusoids. Science 2006; 313: $1287-1290$

25. Janes KA, Albeck JG, Gaudet S, Sorger PK, Lauffenburger DA, Yaffe MB. A systems model of signaling identifies a molecular basis set for cytokine-induced apoptosis. Science 2005; 310: 1646-1653.

26. Beyer EM, MacBeath $\mathrm{G}$. Cross-talk between receptor tyrosine kinase and tumor necrosis factor-alpha signaling networks regulates apoptosis but not proliferation. Mol Cell Proteomics 2012; 11: M111 013292.

27. Hartman ML, Czyz M. Pro-apoptotic activity of $\mathrm{BH} 3$-only proteins and $\mathrm{BH} 3$ mimetics: from theory to potential cancer therapy. Anti-cancer Agents Med Chem 2012; 12: 966-981.

28. Kaushansky A, Ye AS, Austin LS, Mikolajczak SA, Vaughan AM, Camargo N et al. Suppression of host p53 is critical for Plasmodium liver-stage infection. Cell Rep 2013; 3: 630-637.

29. Mele R, Gomez Morales MA, Tosini F, Pozio E. Cryptosporidium parvum at different developmental stages modulates host cell apoptosis in vitro. Infect Immun 2004; 72: 6061-6067.

30. Aoki Mdel P, Cano RC, Pellegrini AV, Tanos T, Guiñazú NL, Coso OA et al. Different signaling pathways are involved in cardiomyocyte survival induced by a trypanosoma cruzi glycoprotein. Microbes Infect 2006; 8: 1723-1731.

31. Guergnon J, Dessauge F, Traincard F, Cayla X, Rebollo A, Bost PE et al. A PKA survival pathway inhibited by DPT-PKI, a new specific cell permeable PKA inhibitor, is induced by T. annulata in parasitized B-lymphocytes. Apoptosis 2006; 11: 1263-1273.

32. Carmen JC, Hardi L, Sinai AP. Toxoplasma gondii inhibits ultraviolet light-induced apoptosis through multiple interactions with the mitochondrion-dependent programmed cell death pathway. Cell Microbiol 2006; 8: 301-315.

(c) (i) (2) (2) Cell Death and Disease is an open-access journal published by Nature Publishing Group. This work is licensed under a Creative Commons Attribution-NonCommercialShareAlike 3.0 Unported License. To view a copy of this license, visit http://creativecommons.org/licenses/by-nc-sa/3.0/ 